\title{
Endoscopic Endoluminal Vacuum Therapy or Self-Expandable Metallic Stent: Treatment Option in Anastomotic Leakage after Esophageal Surgery
}

\author{
Chul-Hyun Lim \\ Department of Internal Medicine, Eunpyeong St. Mary's Hospital, College of Medicine, The Catholic University of Korea, Seoul, Korea
}

See "Endoscopic Management of Anastomotic Leakage after Esophageal Surgery: Ten Year Analysis in a Tertiary University Center" by Nader El-Sourani, Sorin Miftode, Maximilian Bockhorn, Alexander Arlt, Christian Meinhardt, on page 58-66. Clin Endosc 2022;55:41-42

Treatment modalities for esophageal cancer include endoscopic resection, surgery, radiotherapy, chemotherapy, and chemoradiotherapy. Selection of the treatment depends on the stage and physical status of the patient. ${ }^{1}$ Esophagectomy is one of the main curative treatment options in the management of patients with locally advanced esophageal cancer. Despite advances in the surgical techniques and perioperative management, esophagectomy has been reported to be a major procedure associated with a high rate of complications resulting in increased morbidity and mortality rates. ${ }^{2,3}$ Anastomotic leakage is defined as a full-thickness gastrointestinal defect that involves the esophagus, anastomosis, staple line, or conduit. ${ }^{4}$ Recently reported rates of leakage after esophageal cancer surgery range from $10 \%$ to $21.2 \%{ }^{5}$ Several factors, including pulling up of the stomach from the abdominal to the thoracic cavity, limited vascular supply, and mechanical characteristics of the esophagus, contribute to anastomotic leakage after esophageal surgery. Furthermore, patient factors such as

\footnotetext{
Received: November 30, 2021 Revised: December 20, 2021 Accepted: December 22, 2021

Correspondence: Chul-Hyun Lim

Department of Internal Medicine, Eunpyeong St. Mary's Hospital, College of Medicine, The Catholic University of Korea, 1021, Tongil-ro, Eunpyeong-gu, Seoul 03312, Korea

Tel: +82-2-2030-2535, Fax: +82-2-2030-2573, E-mail: diluck@catholic.ac.kr ORCID: https://orcid.org/0000-0002-8347-8979
}

(c) This is an Open Access article distributed under the terms of the Creative Commons Attribution Non-Commercial License (http://creativecommons.org/ licenses/by-nc/3.0) which permits unrestricted non-commercial use, distribution, and reproduction in any medium, provided the original work is properly cited. obesity, heart failure, coronary disease, vascular disease, renal disease, and tobacco use have been reported as risk factors for anastomotic leakage. ${ }^{6}$

The goals for the management of an anastomotic leakage are closure or coverage of the defect, containment of the leak, and drainage of the contaminated space. ${ }^{7}$ Endoscopic and surgical management are the available options for treating anastomotic leakage. Surgical intervention is required in cases of uncontrolled sepsis, failure of initial treatment, and early leakage within the first 72 hours. ${ }^{5}$ Endoscopic management of leakage includes primary closure of defects (clips, endoscopic suturing, and fibrin glue), secondary closure with stents, and endoscopic vacuum therapy (EVT). Primary closure can be applied in early detection of small defects and when the patient's condition is stable; limitations include difficulty in grasping the edge of the defect and poor results if the leak is large (more than $3 \mathrm{~cm}$ ) or asymmetrical. Stenting and EVT can be applied in clinically stable patients with drainage of peri-anastomotic collections. The reported clinical success rates of self-expandable metal stents (SEMS) are up to 74\%; the limitations include potential migration of stents. The clinical success rates of EVT are over $90 \%$ and the limitation includes the need for regular changes in vacuum therapy.

In this issue of Clinical Endoscopy, El-Sourani et al. reported a ten-year experience of endoscopic management of anastomotic leakage after esophageal surgery. ${ }^{8}$ Selection of EVT or SEMS in anastomotic leakage may be difficult for clinicians. There have been few reports analyzing the size of the defect and the presence of a cavity during the endoscopic manage- 
ment of anastomotic leakage after esophageal surgery. They mentioned the defect size and analyzed the potential outcome of the treatment option. They also provided the possibility of applying EVT for treating anastomotic leakage in critically ill patients. EVT was selected in patients with large size defects, high grade in the Surgical Working Group on Endoscopy and Ultrasound classification, and extraluminal cavity, resulting in a complete closure rate of $92.3 \%$ without EVT-related complications. They suggested that SEMS is only warranted in non-septic patients with a small size defect and no extraluminal cavity. The selection of appropriate treatment options for anastomotic leakage after esophageal surgery depends on the characteristics of the defects and the clinical condition of the patients. This study provides practical clues for selecting treatment options for anastomotic leakage after esophageal surgery. Further large-scale studies focusing on the defect size, cavity characteristics, and clinical status of the patients are required for robust conclusions.

Conflict of Interest

The author has no potential conflicts of interest.

Funding

None.

Author Contributions

Writing and approval of final manuscript: Chul-Hyun Lim
ORCID

Chul-Hyun Lim

https://orcid.org/0000-0002-8347-8979

\section{REFERENCES}

1. Kitagawa Y, Uno T, Oyama T, et al. Esophageal cancer practice guidelines 2017 edited by the Japan Esophageal Society: part 1. Esophagus 2019;16:1-24.

2. Dindo D, Demartines N, Clavien PA. Classification of surgical complications: a new proposal with evaluation in a cohort of 6336 patients and results of a survey. Ann Surg 2004;240:205-213.

3. Low DE, Kuppusamy MK, Alderson D, et al. Benchmarking complications associated with esophagectomy. Ann Surg 2019;269:291-298.

4. Low DE, Alderson D, Cecconello I, et al. International consensus on standardization of data collection for complications associated with esophagectomy: Esophagectomy Complications Consensus Group (ECCG). Ann Surg 2015;262:286-294

5. Chevallay M, Jung M, Chon SH, Takeda FR, Akiyama J, Mönig S. Esophageal cancer surgery: review of complications and their management. Ann N Y Acad Sci 2020;1482:146-162.

6. Kassis ES, Kosinski AS, Ross P, Koppes KE, Donahue JM, Daniel VC. Predictors of anastomotic leak after esophagectomy: an analysis of the society of thoracic surgeons general thoracic database. Ann Thorac Surg 2013;96:1919-1926.

7. Schaheen L, Blackmon SH, Nason KS. Optimal approach to the management of intrathoracic esophageal leak following esophagectomy: a systematic review. Am J Surg 2014;208:536-543.

8. El-Sourani N, Miftode S, Bockhorn M, Arlt A, Meinhardt C. Endoscopic management of anastomotic leakage after esophageal surgery - ten year analysis in a tertiary university center. Clin Endosc 2022;55:58-66. 